\title{
CRECIMIENTO, FECUNDIDAD Y DIFERENCIACIÓN SEXUAL DEL LENGUADO Paralichthys adspersus (Steindachner) DE LA COSTA CENTRAL DEL PERÚ
}

\section{GROWTH, FECUNDITY AND SEXUAL DIFFERENTIATION OF THE FINE FLOUNDER Paralichthys adspersus (Steindachner) OF THE CENTRAL COAST OF PERU}

\author{
Beatriz Angeles $^{1}$ y Jaime Mendo ${ }^{2}$
}

\begin{abstract}
Resumen
El lenguado Paralichthys adspersus (Steindachner) es una especie de gran importancia para Chile y el Perú por su abundancia y alto valor comercial, sin embargo muy poco se conoce sobre su potencial de cultivo en el Perú. Este estudio determina el crecimiento y la fecundidad del lenguado y realiza un análisis de caracteres externos que permita su separación por sexos, tres aspectos importantes del cultivo de organismos acuáticos.

Se analizaron 5 caracteres merísticos y 10 morfométricos de 150 ejemplares utilizados para determinar diferencias entre machos y hembras. La edad fue determinada en base a la lectura de anillos en los otolitos, estimando los parámetros de crecimiento de von Bertalanffy mediante el ploteo de Ford-Walford, en tanto la fecundidad se calculó mediante el conteo de ovocitos de gónadas en estadio IV de madurez.

Los caracteres merísticos y morfométricos analizados no permiten separar individuos por sexo, sin embargo, la diferencia observada en cuanto a los orificios anal, urinario y genital presentes en hembras (presenta los 3 separadamente) y machos (sólo 2, anal y urogenital), si permiten realizar dicha separación en forma rápida y confiable.

Las hembras del lenguado presentaron mayor crecimiento que los machos, así la ecuación de von Bertalanffy tiene la forma de: $\mathrm{Lt}=101.17\left(1-\mathrm{e}^{-0.140(\mathrm{t}+0.584)}\right)$ en hembras y $\mathrm{Lt}=60.53\left(1-\mathrm{e}^{-0.253(\mathrm{t}+}\right.$ ${ }^{0.310)}$ ) en machos.

El lenguado es un desovador parcial asincrónico con ovocitos maduros de un diámetro entre 0.665 y $0.805 \mathrm{~mm}$, con una fecundidad total de 2125000 huevos por individuo y una relativa de 1500 huevos por gramo de pez.

Se discute el potencial de esta especie para su cultivo comparando el crecimiento y la fecundidad con otras especies de lenguados de ambiente natural y que se cultivan en el mundo.

Palabras clave: aspectos biológicos, lenguado Paralichthys adspersus, diferenciación sexual, parámetros de crecimiento, fecundidad.
\end{abstract}

Abstract
The fine flounder Paralichthys adspersus (Steindachner) is a species of great importance for Chile and Peru by its abundance and high commercial value, however little is known about its potential for culture in Peru. This study determine growth and fecundity and analyzes external characters, which permit the sex differentiation in order to know about its potential for culture.

Meristic (5) and morphometric (10) characters from 150 individuals were measured and analyzed using linear regressions in order to find differences between sex. Age was determined counting annual rings in otoliths and von Bertalanffy growth parameters were estimated using the FordWalford plot, meanwhile fecundity of females was estimated counting the number of ovocites of mature gonads (stage IV).

The morphometric and meristic characters used in the determination of sexual differentiation, did not show significant differences that can be used in the separation of males from females in a easily and confidentially way. However, it was observed differences in relation to the genital, urinary and anal orifices; females show the 3 orifices separately but males show only two, anal and urogenital orifices.

It was found that $P$. adspersus females growth faster than males. The von Bertalanffy equation for females was $\mathrm{Lt}=101.17\left(1-\mathrm{e}^{-0.140(\mathrm{t}+0.584)}\right)$, and $\mathrm{Lt}=60.53\left(1-\mathrm{e}^{-0.253(\mathrm{t}+0.310)}\right)$ for males.

$P$. adspersus is a partial asynchronic spawner, with mature ovocites between $0.665-0.805 \mathrm{~mm}$. The total fecundity was estimated in 2125000 eggs per individual and the relative fecundity was 1500 eggs per gram of fish.

The potential for culture of this species is discussed comparing growth and fecundity with other flatfish species from natural and cultured stocks. 
Key words: Peru, Paralichthys adspersus, flounder, sexual differentiation, age, growth parameters, fecundity.

\section{Introducción}

El lenguado Paralichthys adspersus (Steindachner, 1867) es una especie de gran importancia en términos de abundancia y valor comercial, cuya distribución abarca desde Paita (Perú) hasta Lota e Islas Juan Fernández (Chile) (Acuña \& Cid, 1995; Chirichigno, 1974).

Su extracción es realizada tanto por embarcaciones artesanales, como por embarcaciones comerciales arrastreras. Los datos de captura proporcionados por el IMARPE y por la FAO para esta especie indican un aumento escalonado de la extracción de esta especie teniendo sus valores más altos a partir de 1984 hasta 1992, reduciéndose significativamente en los años posteriores, lo cual podría deberse a una intensa captura (Chinchayán et al., 1997; Flores et al. 1996, 1997, 1998a, 1998b, 2000a, 2000b) (Figura 1). Esta disminución en las capturas ha incentivado a algunos investigadores y empresas privadas a pensar en la factibilidad de su cultivo, sin embargo es muy poco lo que se conoce sobre su biología y su potencial para el cultivo.

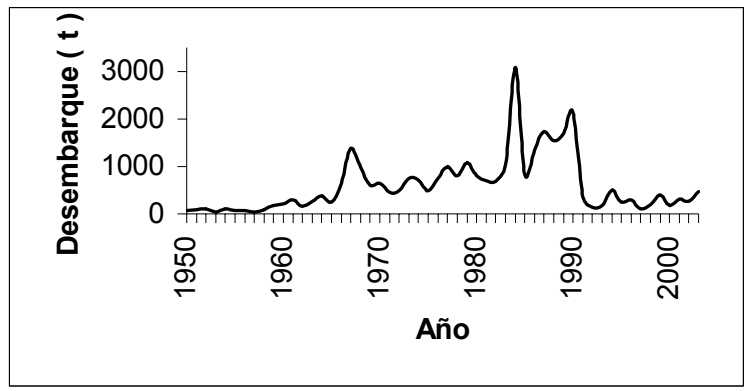

Figura 1. Desembarques de lenguado Paralichthys adspersus en la costa peruana entre 1950 y 2003. (Fuente: 1950-1999 IMARPE, 2000-2003 FAO FIGIS).

En el Perú la escasa información biológica sobre $P$. adspersus, se limita principalmente a algunos aspectos taxonómicos (Chirichigno, 1974) y sobre algunos aspectos biológicos y pesqueros en la zona norte (Samamé \& Castañeda, 1999).

La separación de individuos por sexo, la fecundidad y el crecimiento son aspectos biológicos importantes a ser tomados en cuenta para el cultivo de una especie. En el caso de $P$. adspersus la diferenciación de machos y hembras es difícil de realizar por presentar, aparentemente, características externas muy poco diferenciales. En cuanto a la información existente sobre edad y crecimiento, ésta se limita a la zona norte del país, y en cuanto a la fecundidad no existe información publicada al respecto. El presente trabajo tiene como objetivo evaluar el potencial de crecimiento y reproductivo de Paralichthys adspersus de la costa central del Perú, a través de la estimación de parámetros de crecimiento, y la determinación de la fecundidad total de esta especie, que sirva como base para determinar la factibilidad de cultivo de esta especie. Asimismo siendo la separación de individuos hembras y machos en los estudios de dinámica poblacional y cultivo, se trata de identificar caracteres secundarios externos, morfométricos o merísticos que faciliten la separación de individuos por sexo.

\section{Materiales y métodos}

El material biológico para la determinación de diferenciación sexual, la edad y la fecundidad total, consistió en un total de 150 individuos, que fueron colectados en los puertos de Ancón (11 ${ }^{\circ} 44^{\prime} \mathrm{S}$; $\left.77^{\circ} 12^{\prime} \mathrm{W}\right)$, Callao $\left(12^{\circ} 03^{\prime} \mathrm{S} ; 7^{\circ} 09^{\prime} \mathrm{W}\right)$, Chorrillos $\left(12^{\circ} 09.5^{\prime} \mathrm{S} ; \quad 77^{\circ} 01.5^{\prime} \mathrm{W}\right)$ y Pucusana $\left(12^{\circ} 28^{\prime} \mathrm{S}\right.$; $76^{\circ} 47.8^{\prime} \mathrm{W}$ ) (Figura 2) durante Setiembre 1993 a Junio 1994, considerada como la época de mayor actividad reproductiva de esta especie (Acuña \& Cid, 1995; Samamé \& Castañeda, 1999).

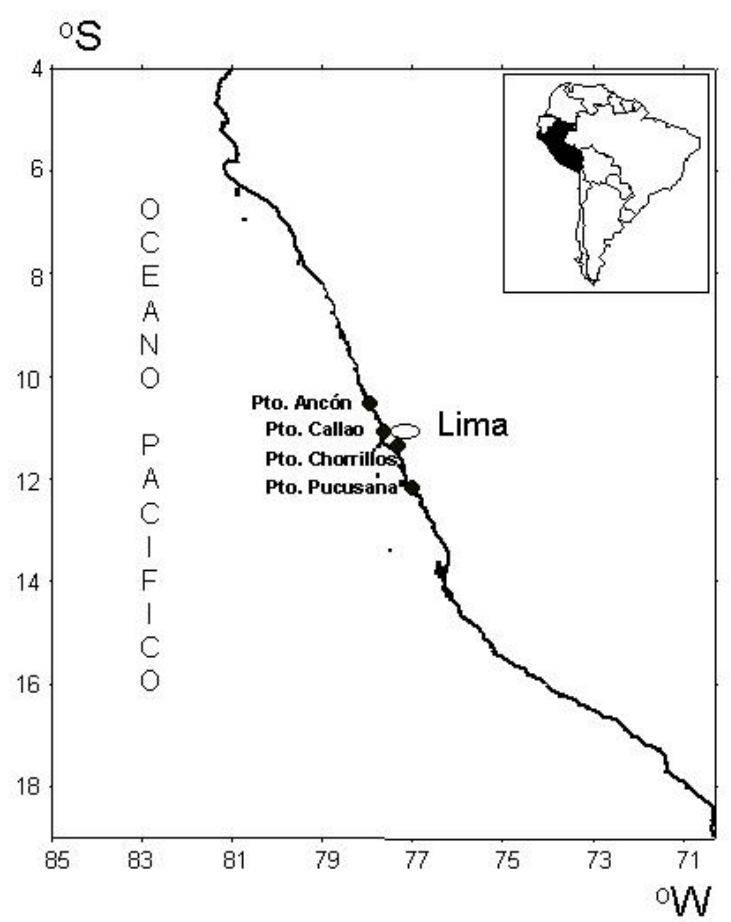

Figura 2. Ubicación de los lugares de muestreo de $P$. adspersus.

En el laboratorio los lenguados fueron medidos, pesados, y se anotaron 5 caracteres merísticos y 10 morfométricos con el fin de determinar diferencias 
sexuales (Figura 3). Para la obtención de datos merísticos se procedió al conteo del número de radios de las aletas dorsal (RAD), pectoral (RAP) y anal (RAA), así como del número de branquiespinas epibranquiales (BRE) y ceratobranquiales (BRC). Para determinar la existencia de diferencias entre machos y hembras en las variables merísticas y morfométricas empleadas, se realizaron análisis de correlación y de comparación de regresiones lineales. Asimismo se empleó el análisis de varianza (ANVA) para comparar las medias de los caracteres merísticos y morfométricos de hembras y machos (Zar, 1984). Adicionalmente se observó cuidadosamente la anatomía externa para la identificación de algún carácter diferencial entre hembras y machos.

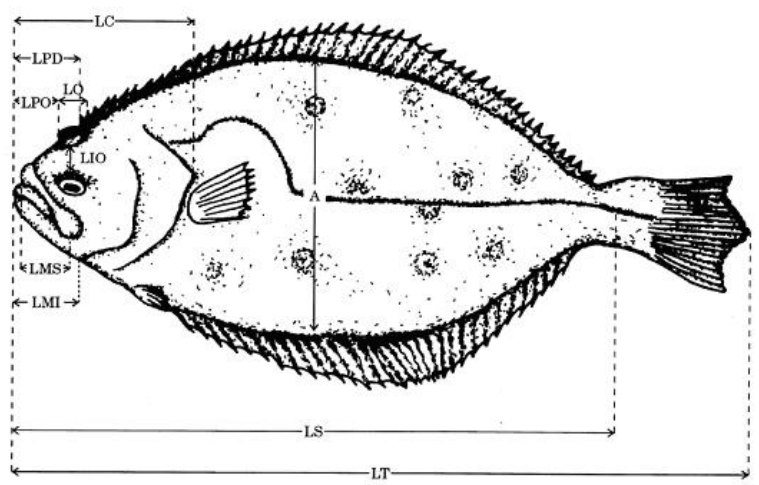

Figura 3. Caracteres morfométricos tomados en cada ejemplar de $P$. adspersus (LT= longitud total, $\mathrm{LS}=$ longitud Standard, $\mathrm{LC}=$ longitud de la cabeza, $\mathrm{A}=$ altura, LMS $=$ longitud de la mandíbula superior, $\mathrm{LMI}=$ longitud de la mandíbula inferior, $\mathrm{LIO}=$ longitud interorbital, $\mathrm{LO}=$ longitud orbital, $\mathrm{LPD}=$ longitud predorsal).

La edad fue determinada en base a la lectura de los otolitos (sagitta), los cuales fueron observados en un estereoscopio siendo sumergidos en glicerina sobre fondo negro y con luz dirigida desde arriba. La lectura de la edad se realizó en el otolito derecho por la cara externa y hacia la parte anterior, determinando el tiempo de formación de los anillos mediante el examen de la variación mensual en las dimensiones del borde hialino u opaco. El método usado para el cálculo de las longitudes medias por edad fue el retrocálculo, para lo cual se registró, con ayuda del ocular micrométrico, el radio del otolito $(\mathrm{R})$ y el radio de cada anillo (ri), y se estableció posteriormente la relación entre la longitud del pez (L) y el radio (R) (Sparre et al., 1989) (Figura 4). La curva de crecimiento en longitud y peso fue ajustada a la ecuación de von Bertalanffy:

$$
\mathrm{L}_{\mathrm{t}}=\mathrm{L}_{\infty}\left(1-\mathrm{e}^{-\mathrm{K}\left(\mathrm{t}-\mathrm{t}_{\mathrm{o}}\right)}\right) \text { y } \mathrm{P}_{\mathrm{t}}=\mathrm{P}_{\infty}\left(1-\mathrm{e}^{-\mathrm{K}\left(\mathrm{t}-\mathrm{t}_{\mathrm{o}}\right)}\right)
$$
donde:

$\mathrm{L}_{\mathrm{t}}$ y $\mathrm{P}_{\mathrm{t}}=$ longitud y peso a la edad $\mathrm{t}$, respectivamente,
$\mathrm{K}=$ constante de crecimiento,

$\mathrm{L}_{\infty}$ y $\mathrm{P}_{\infty}=$ longitud y peso asintótico, respectivamente, $\mathrm{t}_{\mathrm{o}}=$ edad a la longitud 0 ,

$\mathrm{t}=$ edad

Los parámetros $\mathrm{L}_{\infty}, \mathrm{P}_{\infty}$ y $\mathrm{K}$ fueron estimados mediante el ploteo de Ford-Walford : $\mathrm{L}_{\mathrm{t}+1}=\mathrm{a}+\mathrm{b} \mathrm{L}_{\mathrm{t}}$

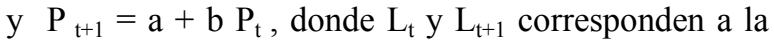
longitud separada por un intervalo constante de tiempo. De esto se deriva: $\mathrm{L}_{\infty}=\mathrm{a} /(1-b)$ y $K=-\ln \mathrm{b}$. La estimación de $t_{0}$ se llevó a cabo despejando $t_{o}$ de la ecuación de von Bertalanffy (Sparre et al., 1989):

$$
\mathrm{t}_{\mathrm{o}}=\mathrm{t}_{1}+\frac{1}{\mathrm{~K}} \ln \left(1-\frac{\mathrm{L}_{(\mathrm{t} 1)}}{\mathrm{L}_{\infty}}\right)
$$

Con los parámetros de la fórmula de von Bertalanffy se determinó el índice de rendimiento en crecimiento $\left(\phi^{\prime}\right)$ para ejemplares machos y hembras: $\phi^{\prime}=\log \mathrm{K}+2 \log \mathrm{L}_{\infty}$, con el fin de comparar el crecimiento del lenguado común con otros peces planos ya que la comparación en base a $\mathrm{K}$ y $\mathrm{L}_{\infty}$ separadamente no es recomendable (Pauly \& Munro, 1984).

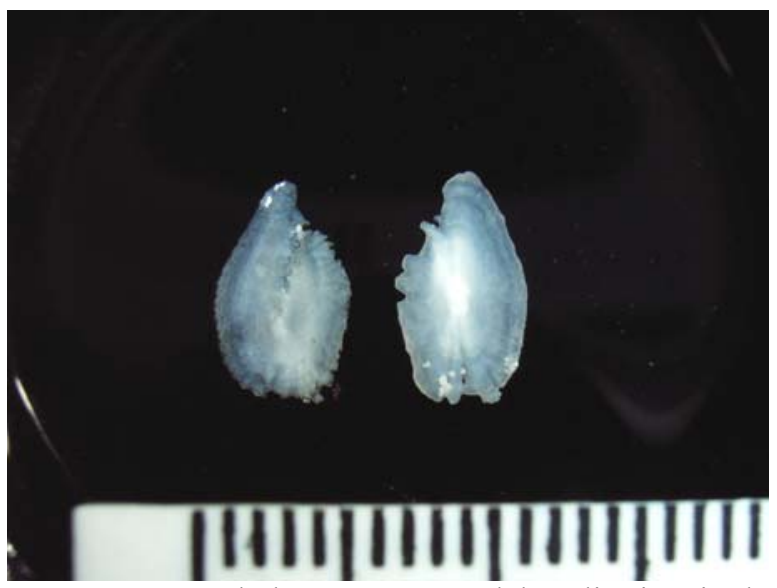

Figura 4. Foto de la cara externa del otolito izquierdo y derecho de P.adspersus indicando las mediciones tomadas para este estudio $(\mathrm{D}=$ diámetro, $\mathrm{A}=$ ancho, $\mathrm{R}=$ radio, $\mathrm{r}_{1,2}=$ anillos de crecimiento).

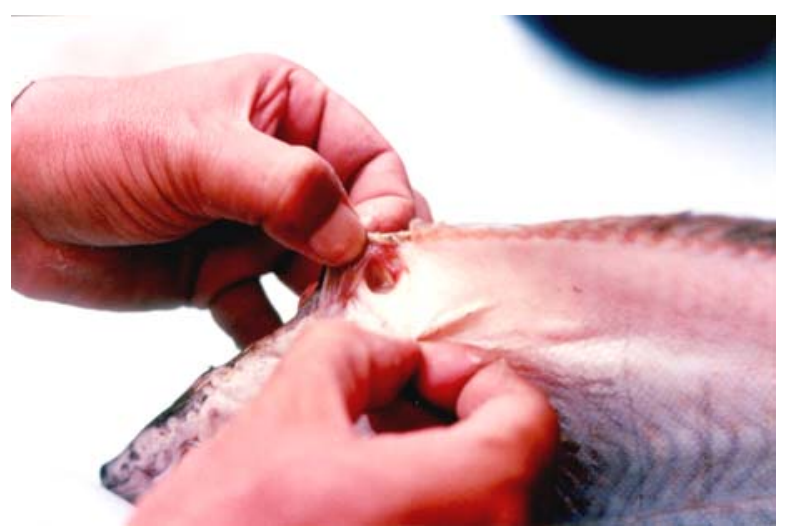

Figura 5. Foto orificio genital en hembras de lenguado $P$. adspersus. 
En la determinación de la fecundidad total se emplearon las gónadas de hembras en estadío IV de madurez definidas por Ramos (1982) como gónadas con ovocitos visibles, opacos de diferentes dimensiones y algunos transparentes sueltos, con coloración anaranjada. Estas fueron colocadas independientemente en medio Gilson para la liberación de los ovocitos del tejido ovárico (Jones, 1974). Los ovocitos liberados fueron tamizados a 150 $\mu \mathrm{m}$, descartando aquellos menores a esta talla dado que aún no habían entrado a la segunda fase de crecimiento, pudiendo ser reabsorbidos; posteriormente fueron colocados en un vaso de precipitados y aforados a 1 litro con agua corriente. Esta solución se mantuvo en constante agitación, para la toma de 3 muestras de $1 \mathrm{ml}$. Los ovocitos colectados fueron colocados en una cámara Newbauer bajo estereoscopio a $3 \mathrm{X}$, para su conteo y medición, promediando posteriormente los valores para obtener la fecundidad total y la distribución de frecuencias de tallas de ovocitos de cada gónada.

\section{Resultados}

Diferenciación sexual

El análisis de correlación múltiple nos muestra que tanto en hembras como en machos todas las variables originales están altamente correlacionadas, mientras que las variables estandarizadas con respecto a la longitud estándar (LS) muestran algunas correlaciones poco significativas (Tablas 1 y 2). Las mejores correlaciones de las variables estandarizadas se dieron entre la longitud de la mandíbula superior (LMS), e inferior $(\mathrm{r}=0.779$ para hembras y $\mathrm{r}=0.614$ para machos), la longitud de la mandíbula inferior (LMI) y la longitud preorbital (LPO) $(\mathrm{r}=0.690$ hembras y $\mathrm{r}=$ 0.626 machos), la longitud interorbital (LIO) y el diámetro orbital $(\mathrm{DO})(\mathrm{r}=-0.513$ hembras y $\mathrm{r}=-0.783$ machos).

La comparación entre las regresiones lineales de los caracteres morfométricos originales con respecto a la LS, para hembras y machos, presentó diferencias significativas para algunos caracteres como la longitud interorbital (LIO), la longitud orbital (LO), y la longitud predorsal (LPD), en tanto los caracteres merísticos presentaron diferencias significativas para el número de radios de la aleta anal (RAA), sin embargo los análisis de correlación y de varianza nos muestran que una separación de individuos por sexo en base a estos caracteres es imposible (Tablas 3 y 4).

La evaluación de características externas permitió la identificación de un carácter anatómico externo diferencial para hembras y machos con respecto a los orificios anal, urinario y genital. Los individuos machos solo presentan dos orificios (anal y el urogenital) en tanto las hembras presentaron los 3 orificios separadamente (Figura 5). Esta diferencia en el número de orificios permite realizar una separación confiable de individuos por sexo.
Tabla 1. Matriz de coeficientes de correlación entre los caracteres morfométricos de hembras de $P$. adspersus. Sobre la diagonal: datos originales, bajo la diagonal: datos estandarizados a la LS. $\left(\mathrm{r}_{0.05(70)}=0.232 ; \mathrm{r}_{0.01(70)}=0.307\right)$.

\begin{tabular}{|c|cccccccccc|}
\hline & LT & LS & A & LC & LMS & LMI & LPO & LIO & DO & LPD \\
\hline LT & 1 & 0.998 & 0.989 & 0.991 & 0.991 & 0.992 & 0.983 & 0.947 & 0.920 & 0.961 \\
LS & - & 1 & 0.989 & 0.988 & 0.987 & 0.990 & 0.978 & 0.938 & 0.924 & 0.956 \\
A & - & - & 1 & 0.984 & 0.976 & 0.982 & 0.969 & 0.948 & 0.921 & 0.938 \\
LC & - & - & 0.172 & 1 & 0.991 & 0.995 & 0.992 & 0.966 & 0.918 & 0.973 \\
LMS & - & - & 0.042 & 0.400 & 1 & 0.992 & 0.988 & 0.952 & 0.925 & 0.968 \\
LMI & - & - & 0.116 & 0.484 & 0.779 & 1 & 0.988 & 0.951 & 0.919 & 0.968 \\
LPO & - & - & 0.204 & 0.293 & 0.687 & 0.690 & 1 & 0.960 & 0.912 & 0.978 \\
LIO & - & - & 0.547 & 0.162 & 0.196 & 0.104 & 0.413 & 1 & 0.872 & 0.937 \\
DO & - & - & -0.196 & 0.190 & 0.397 & 0.406 & 0.087 & -0.512 & 1 & 0.890 \\
LPD & - & - & -0.151 & 0.318 & 0.603 & 0.645 & 0.592 & 0.065 & 0.416 & 1 \\
\hline
\end{tabular}

Tabla 2. Matriz de coeficientes de correlación entre los caracteres morfométricos de machos de $P$. adspersus. Sobre la diagonal: datos originales, bajo la diagonal: datos estandarizados a la LS. $\left(\mathrm{r}_{0.05(57)}=0.256 ; \mathrm{r}_{0.01(57)}=0.333\right)$.

\begin{tabular}{|c|cccccccccc|}
\hline \multicolumn{2}{|c}{ LT } & LS & A & LC & LMS & LMI & LPO & LIO & DO & LPD \\
\hline LT & 1 & 0.998 & 0.993 & 0.995 & 0.992 & 0.993 & 0.989 & 0.969 & 0.962 & 0.70 \\
LS & - & 1 & 0.992 & 0.993 & 0.992 & 0.991 & 0.987 & 0.965 & 0.967 & 0.69 \\
A & - & - & 1 & 0.990 & 0.986 & 0.986 & 0.983 & 0.972 & 0.959 & 0.71 \\
LC & - & - & -0.132 & 1 & 0.991 & 0.994 & 0.992 & 0.974 & 0.960 & 0.75 \\
LMS & - & - & -0.322 & 0.465 & 1 & 0.992 & 0.987 & 0.964 & 0.956 & 0.68 \\
LMI & - & - & -0.231 & 0.727 & 0.614 & 1 & 0.991 & 0.972 & 0.954 & 0.72 \\
LPO & - & - & -0.041 & 0.622 & 0.456 & 0.627 & 1 & 0.973 & 0.944 & 0.75 \\
LIO & - & - & 0.640 & -0.222 & -0.366 & -0.267 & 0.010 & 1 & 0.913 & 0.51 \\
DO & - & - & -0.469 & 0.483 & 0.464 & 0.495 & 0.193 & -0.783 & 1 & 0.40 \\
LPD & - & - & -0.084 & 0.610 & 0.354 & 0.526 & 0.527 & -0.278 & 0.463 & 1 \\
\hline
\end{tabular}

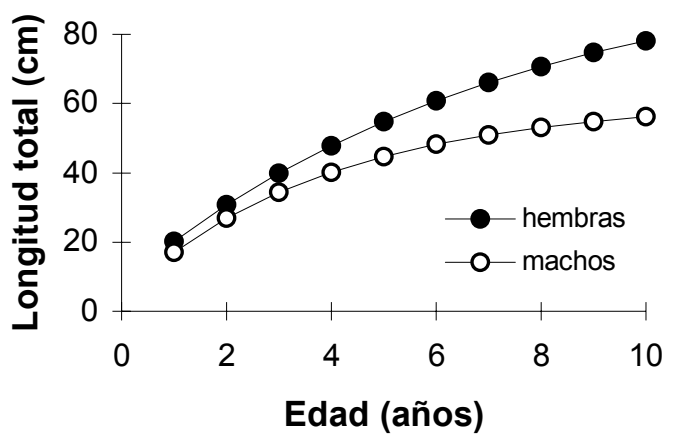

Figura 6. Datos de edad y longitud de P. adspersus estimados en base a la ecuación de von Bertalanffy.

\section{Crecimiento}

El patrón de formación de anillos en el lenguado común muestra una zona opaca y una hialina por año, la cual ocurre durante los meses de verano e invierno respectivamente. La relación entre el radio del otolito ( $\mathrm{R}$, en micras) y la longitud del pez $(\mathrm{L}, \mathrm{cm})$ obtenida 
para calcular la longitud por anillo de crecimiento obedece a la ecuación:

$\mathrm{L}=-7.378+1.464 \mathrm{R}$ para hembras $(\mathrm{n}=66) \mathrm{y}$

$\mathrm{L}=-4.978+1.261 \mathrm{R}$ para machos $(\mathrm{n}=54)$

En la Tabla 3 se presentan las longitudes medias por anillo de crecimiento para individuos machos y hembras, e indica un mayor crecimiento de las hembras con respecto a los machos, tal como ocurre en la mayoría de peces planos.

Tabla 3. Media, desviación standard (ds) y valores de F de los caracteres morfométricos ( $\%$ de la longitud standard) de $P$. adspersus. Donde: A= altura, $\mathrm{LC}=$ Longitud de la cabeza, LMS $=$ Longitud mandíbula superior, LMI=longitud mandíbula inferior, $\mathrm{LPO}=$ Longitud preorbital, $\mathrm{LIO}=$ Longitud interorbital, $\mathrm{LO}=$ longitud orbital, $\mathrm{LPD}=$ longitud predorsal

\begin{tabular}{|c|c|c|c|c|c|}
\hline \multirow{2}{*}{ Caracter } & \multicolumn{2}{|c|}{ Hembras } & \multicolumn{2}{c|}{ Machos } & \multirow{2}{*}{$\begin{array}{c}\text { Valores } \\
\end{array}$} \\
\cline { 2 - 5 } & media & $\mathrm{ds}$ & media & $\mathrm{ds}$ & de F \\
\hline A & 44.95 & 2.085 & 45.08 & 2.228 & 0.1333 \\
LC & 27.36 & 3.635 & 28.17 & 1.146 & 2.6571 \\
LMS & 12.67 & 0.639 & 12.51 & 0.550 & 2.3544 \\
LMI & 15.18 & 0.683 & 15.10 & 0.681 & 0.3713 \\
LPO & 6.25 & 0.416 & 6.26 & 0.312 & 0.0342 \\
LIO & 2.41 & 0.517 & 2.14 & 0.474 & $9.3432^{*}$ \\
LO & 5.48 & 0.795 & 6.20 & 0.687 & $30.817^{*}$ \\
LPD & 6.74 & 0.620 & 7.05 & 0.562 & $8.7305^{*}$ \\
\hline
\end{tabular}

* Significativo para $\mathrm{P}=0.05$

Tabla 4. Rango, promedio (x) y desviación standard (ds) de los caracteres merísticos de $P$. adspersus y valores de $\mathrm{F}$ obtenidos de la prueba de ANVA. Donde: $\mathrm{RAD}=$ número de radios aleta dorsal, $\mathrm{RAA}=$ número de radios aleta anal, $\mathrm{RAP}=$ número de radios de la aleta pectoral, $\mathrm{BRE}=$ branquiespinas epibranquiales, $\mathrm{BRC}=$ branquiespinas ceratobranquiales.

\begin{tabular}{|l|c|ccccc|}
\hline \multirow{2}{*}{ Muestra } & Parámetros & \multicolumn{5}{|c|}{ Caracteres Merísticos } \\
\cline { 3 - 7 } & & RAD & RAA & RAP & BRE & BRC \\
\hline \multirow{3}{*}{ Hembras } & rango & $65-76$ & $52-61$ & $10-13$ & $6-9$ & $15-19$ \\
& $\overline{\mathrm{x}}$ & 70.79 & 56.69 & 12.22 & 7.02 & 17.27 \\
& $\mathrm{ds}$ & 2.390 & 2.148 & 0.566 & 0.655 & 0.938 \\
\hline \multirow{3}{*}{ Machos } & rango & $67-77$ & $52-60$ & $11-13$ & $6-9$ & $15-19$ \\
& $\overline{\mathrm{x}}-$ & 70.345 & 55.55 & 12.10 & 7.09 & 17.38 \\
& $\mathrm{ds}$ & 2.107 & 1.808 & 0.447 & 0.674 & 0.971 \\
\hline Valores de F & & 1.126 & 8.473 & 1.145 & 0.451 & 0.319 \\
\hline Nivel de & & n.s. & $* *$ & n.s. & n.s. & n.s. \\
significancia & & & & & & \\
\hline
\end{tabular}

** Significativo para $\mathrm{P}=0.01$

Los parámetros de crecimiento de la ecuación de von Bertalanffy obtenidos mediante el ploteo de FordWalford, empleando la longitud media por edad, se presentan en la Tabla 4.

De esta manera la ecuación de crecimiento en longitud de von Bertalanffy para el lenguado $P$. adspersus toma la forma de: $\mathrm{L}_{\mathrm{t}}=101.169\left(1-\mathrm{e}^{-0.139(\mathrm{t}+}\right.$ ${ }^{0.584)}$ ) para hembras y de $\mathrm{L}_{\mathrm{t}}=60.539\left(1-\mathrm{e}^{-0.253(\mathrm{t}+0.310)}\right.$ ) para machos; en tanto para el peso se tiene: $\mathrm{P}_{\mathrm{t}}=16$ 412.72(1- $\left.\mathrm{e}^{-0.139(\mathrm{t}+0.584)}\right)^{3.27}$ para hembras, y $\mathrm{P}_{\mathrm{t}}=3$ $145.54\left(1-\mathrm{e}^{-0.253(\mathrm{t}+0.310)}\right)^{3.27}$ para machos.

Como puede observarse en la Figura 6 las hembras y machos de lenguado común ( $P$. adspersus) alcanzan talla y peso comercial $(40-47 \mathrm{~cm}, 770-1400 \mathrm{~g})$, en el medio natural, entre los 3 y 4 años, con una ligera ventaja de las hembras con respecto a los machos. Esta diferencia se acrecienta a partir del cuarto año, para finalmente establecerse un dimorfismo sexual en crecimiento muy marcado.

Tabla 5. Valores promedio de los radios $\left(r_{i}\right)$ de cada zona de crecimiento en los otolitos de $P$. adspersus.

\begin{tabular}{|c|c|c|c|c|c|c|}
\hline \multirow{2}{*}{ ANILLO } & \multicolumn{3}{|l}{ HEMBRAS } & \multicolumn{4}{|c|}{ MACHOS } \\
\cline { 2 - 7 } & $\mathrm{n}$ & $\mathrm{r}_{\mathrm{i}}$ & $\mathrm{ds}$ & $\mathrm{n}$ & $\mathrm{r}_{\mathrm{i}}$ & $\mathrm{ds}$ \\
\cline { 1 - 7 } Demersal & 50 & 10.76 & 0.981 & 41 & 10.85 & 1.174 \\
1 & 66 & 18.64 & 2.146 & 53 & 18.49 & 2.035 \\
2 & 60 & 25.30 & 3.619 & 41 & 25.68 & 1.968 \\
3 & 44 & 32.25 & 2.081 & 29 & 31.14 & 1.642 \\
4 & 20 & 38.20 & 1.399 & 7 & 35.43 & 0.976 \\
5 & 10 & 42.55 & 1.383 & 4 & 39.25 & 0.957 \\
6 & 4 & 46.50 & 1.291 & 2 & 41.50 & 0.707 \\
\hline
\end{tabular}

Fecundidad

La distribución de frecuencias de tallas de ovocitos en los ovarios de lenguados en diferentes estados de madurez, muestra diferentes grupos modales (Figura 7), indicando que $P$. adspersus es un desovador parcial con ovocitos maduros de un diámetro entre 0.665 y $0.805 \mathrm{~mm}$. Su fecundidad total promedio es de 2125000 , con un promedio de 1500 huevos por gramo de pez (Figura 8).

\section{Discusión}

Los resultados obtenidos en el presente estudio muestran que no es posible separar machos y hembras de $P$. adspersus en base a los caracteres merísticos y morfométricos como se ha practicado en Solea solea (Ramos \& Ramos, 1985), ni en base a otros caracteres sexuales secundarios accesorios tales como coloración, reportado en el caso de Arapaima gigas (Copaira \& Montalvo, 1972), abultamiento en la zona de la nuca en Aequidens rivulatus o transformaciones en las mandíbulas en los machos Oncorhynchus sp. y Salmo sp. (Tresierra et al., 1984). Sin embargo, los individuos machos solo presentan dos orificios (anal y el urogenital) en tanto las hembras presentaron los 3 orificios separadamente, lo que permite realizar dicha separación (Figura 5), tal como se practica en tilapias (Huet, 1983). 
Tabla 6. Longitudes $(\mathrm{cm})$ por edad de $P$. adspersus calculados mediante la relación radio del otolito-longitud total. Donde: $\mathrm{N}=$ individuos que presentaron esa edad, $\mathrm{LT} / \mathrm{R}=$ longitud total calculada en base al radio del otolito.

\begin{tabular}{|c|c|c|c|c|}
\hline \multirow{2}{*}{$\begin{array}{c}\text { Edad } \\
\text { (años) }\end{array}$} & \multicolumn{2}{|c|}{ Hembras } & \multicolumn{2}{c|}{ Machos } \\
\cline { 2 - 5 } & $\mathrm{N}$ & LT/R & $\mathrm{N}$ & LT/R \\
\hline 1 & 66 & 19.91 & 53 & 18.34 \\
2 & 60 & 29.66 & 41 & 27.40 \\
3 & 44 & 39.84 & 29 & 34.28 \\
4 & 20 & 48.55 & 7 & 39.69 \\
5 & 10 & 54.92 & 4 & 44.51 \\
6 & 4 & 60.70 & 2 & 47.35 \\
\hline
\end{tabular}

Tabla 7. Parámetros de crecimiento y indice de rendimiento en crecimiento de 4 especies del género Paralichthys.

\begin{tabular}{|c|c|c|c|c|c|c|}
\hline Especie & $\begin{array}{c}\text { LT max. } \\
(\mathrm{cm})\end{array}$ & $\mathbf{L}_{\infty}$ & K & $t_{0}$ & $\phi^{\prime}$ & Sexo \\
\hline $\begin{array}{l}\text { Paralichthys } \\
\text { olivaceus }{ }^{I}\end{array}$ & 103 & 117 (SL) & 0.150 & & 3.31 & \\
\hline$P$. dentatus ${ }^{I}$ & 94 & $85.9(\mathrm{TL})$ & 0.215 & & 3.20 & \\
\hline P. californicus ${ }^{I}$ & 152 & $137(\mathrm{TL})$ & 0.08 & 1.2 & 3.18 & $\mathrm{H}$ \\
\hline & 152 & $148(\mathrm{TL})$ & 0.1 & 0.2 & 3.34 & $\mathrm{H}$ \\
\hline P. adspersus ${ }^{2}$ & 84 & 87.8 & 0.2 & 0.46 & 3.188 & \\
\hline
\end{tabular}

Fuente: ${ }^{1}$ FISHBASE, ${ }^{2}$ Samamé \& Castañeda (1999)

La determinación del tiempo de formación de la zona hialina y opaca en los otolitos permite establecer si éstas se forman una o más veces por año (Mendo, 1987). En el caso de $P$ adspersus el patrón de formación de anillos con una zona opaca en verano y una hialina en invierno por año, concuerda con lo reportado para especies pelágicas y demersales de la costa peruana tales como la sardina (Sardinops sagax sagax) y la cachema (Cynoscion analis) (Mendo, 1987).

Los análisis realizados en este estudio permiten comprobar que esta especie presenta marcadas diferencias en el crecimiento de hembras y machos, las cuales se acentúan a partir del 4to año de vida. Resultados similares son encontrados por Samamé y Castañeda (1999), quienes determinaron que en ejemplares del lenguado $P$. adspersus de la zona norte de Perú, es posible apreciar dichas diferencias en crecimiento desde los $40 \mathrm{~cm}$, longitud que alcanzarían entre el 3er y 4to año de vida. Asimismo, los parámetros de crecimiento obtenidos en este estudio difieren de los reportados por Samamé \& Castañeda (1999) para la misma especie en la costa norte del litoral peruano. Dichos autores realizan el cálculo de los parámetros de crecimiento en forma conjunta para hembras y machos, por no contar con un amplio rango en la distribución de edades de los machos, sin embargo, dado que el número de hembras analizadas es casi el triple de los machos (hembras $=92$, machos $=36)$, los resultados de su ecuación $\left(\mathrm{L}_{\infty}=87.8\right.$ $\mathrm{cm}, \mathrm{K}=0.2, \mathrm{t}_{\mathrm{o}}=0.46$ y $\mathrm{P}_{\infty}=9118 \mathrm{~g}$ ) tienden $\mathrm{a}$ presentar valores más cercanos a los encontrados para las hembras en este estudio. Por consiguiente el índice de rendimiento en crecimiento que se puede obtener de sus datos de crecimiento $\left(\phi^{\prime}=3.188\right)$ es similar al obtenido en este estudio para las hembras $\left(\phi^{\prime}=3.155\right)$. Es importante mencionar que las diferencias en edad y crecimiento entre machos y hembras registrados en el presente estudio, hace difícil una comparación más detallada con los resultados obtenidos por Samamé \& Castañeda (1999).

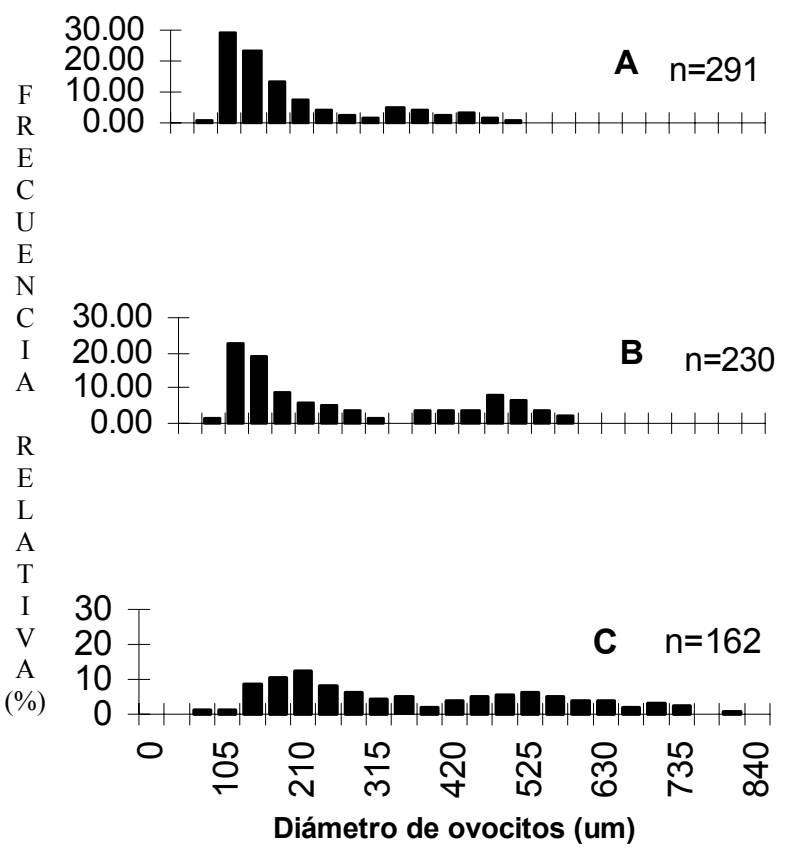

Figura 7. Histograma de la frecuencia relativa del tamaño de los ovocitos de $P$. adspersus con respecto a su diámetro, en estadíos de madurante inicial (A), intermedio (B) y en desove (C).

Si comparamos los parámetros de crecimiento obtenidos en este estudio con los de otras especies del mismo genero como $P$. olivaceus, $P$. californicus y $P$. dentatus, encontramos diferencias notorias; sin embargo los valores del índice de rendimiento en crecimiento $\left(\phi^{\prime}\right)$ de estas especies (entre 3.18 y 3.34 ) es similar al encontrado para las hembras (3.155) y ligeramente mayor que el de los machos (2.968) de este estudio (Tabla 7). Cabe mencionar que en estos estudios no se reporta el sexo de los ejemplares analizados. Los valores de longitud total máxima reportados para estas especies $(84-152 \mathrm{~cm})$ sobrepasan los valores de longitud infinita calculados para hembras y machos de $P$. adspersus en la costa peruana. 

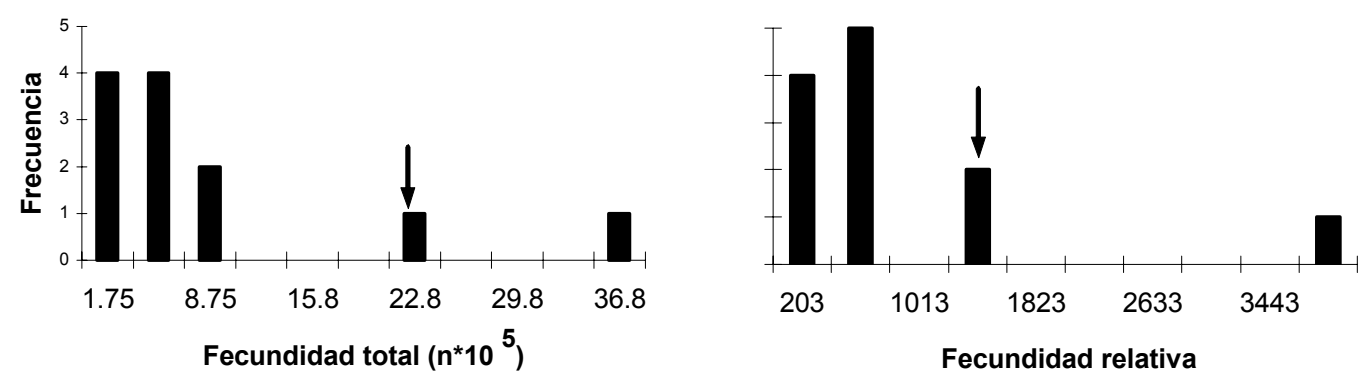

Figura 8. Distribución de fecundidad total y fecundidad relativa de 12 especies de peces planos. La flecha indica el valor para $P$. adspersus.

El diámetro de los ovocitos maduros registrados en este estudio para $P$. adspersus no difieren de los obtenidos para otras especies del género Paralichthyidae ( $P$. microps $(0.68-0.85 \mathrm{~mm}), \quad P$. californicus $(0.74-0.82 \mathrm{~mm}), P$. woolmani $(0.82-0.90$ $\mathrm{mm})$ ), en tanto que son ligeramente menores a Solea solea $(0.98-1.58 \mathrm{~mm})$ y Scophthalmus maximus $(0.9$ $1.2 \mathrm{~mm}$ ) (Ahlstrom et al., 1984; Silva, 1990, 1994; Futagawa com. pers.). Los datos de fecundidad proporcionados por la literatura para los peces planos muestran una gran variabilidad específica, la cual está relacionada con las características de los individuos muestreados y el método de estimación empleado. $\mathrm{Si}$ comparamos la fecundidad total estimada para $P$. adspersus (2 124766 huevos por individuo), con la de Scophthalmus maximus (3 658 740) (Jones, 1974) у Solea solea (425 000) (Ramos, 1982), encontramos que el lenguado presenta una fecundidad muy interesante desde el punto de vista de la producción, habiéndose demostrado que su fecundación en sistemas de cultivo es viable, y es posible obtener larvas y juveniles en un promedio de 2 meses y un nivel de sobrevivencia de 24\% (Silva, 2001; Silva \& Flores, 1989).

Samamé \& Castañeda (1999) reportan que lo machos inician la evacuación de productos sexuales a una talla de $34 \mathrm{~cm}$ de longitud con una talla de primer desove de $43.1 \mathrm{~cm}$, en tanto que las hembras iniciarían la evacuación de óvulos a los $50 \mathrm{~cm}$, siendo la talla de primer desove los $60.4 \mathrm{~cm}$. Teniendo en cuenta esta información y basados en los datos de crecimiento de este estudio, encontramos que el inicio de la reproducción en machos de $P$. adspersus se daría a partir de los 3 años, en tanto que en hembras se hallaría entre el 4to y 5to año, información que debería tenerse en cuenta en los planes de manejo de esta especie.

Si bien en el medio natural el lenguado $P$. adspersus alcanzaría talla comercial entre los 3 y 4 años $(770$ - 1400 gr) las experiencias de cultivo realizadas a la fecha indican que alcanzaría su talla comercial (1000 gr) en 2.8 años (Silva, 2001). La producción sucesiva de ejemplares adaptados al

cautiverio, así como la tecnificación de su cultivo podría contribuir al incremento de las tasas de crecimiento, tal como ocurrió con el turbot (Scophthalmus maximus) que en la década del 90 requería de un periodo de 4 años ( 1 año de nursery +3 de engorde) para alcanzar 1000g (Coll, 1991), y actualmente poco más de dos años $(25$ meses) (Vásquez et al., 2002).

En líneas generales podemos indicar que esta es una especie con tasas de crecimiento similares a otros peces planos, con diferencias marcadas entre hembras y machos, siendo las hembras las que alcanzan los mayores valores de talla y peso, con diferencias sexuales reconocibles $y$ un alto potencial de fecundidad.

\section{Conclusiones}

- Los caracteres merísticos y morfométricos empleados para la determinación de diferencias sexuales en el lenguado Paralichthys adspersus no mostraron diferencias que permitieran separar individuos por sexo en forma rápida y confiable.

- Los individuos machos solo presentan dos orificios que corresponde al anal y el urogenital y las hembras presentaron los 3 orificios separadamente. Esta diferencia en el número de orificios permite realizar una separación confiable de individuos por sexo.

- Las hembras y los machos presentaron diferencias significativas en el crecimiento, siendo las hembras las que presentan un mayor crecimiento.

- La ecuación de von Bertalanffy tiene la forma de: $\mathrm{Lt}=101.17\left(1-\mathrm{e}^{-0.140}(\mathrm{t}+0.584)\right.$ en hembras $\mathrm{y}$ $\mathrm{Lt}=60.53\left(1-\mathrm{e}^{-0.253(t+0.310)}\right)$ en machos, en tanto que el índice de rendimiento en crecimiento $\left(\phi^{\prime}\right)$ presenta un valor de 3.155 para las hembras y de 2.968 para los machos.

- El lenguado P. adspersus es un desovador parcial asincrónico con ovocitos maduros de un diámetro entre 0.665 y $0.805 \mathrm{~mm}$, con una fecundidad total de 2125000 huevos por individuo y una relativa de 1500 huevos por gramo de pez. 
- En líneas generales podemos indicar que esta es una especie con tasas de crecimiento similares a otros peces planos, con diferencias marcadas entre hembras y machos, siendo las hembras las que alcanzan los mayores valores de talla y peso, con diferencias sexuales reconocibles $y$ un alto potencial de fecundidad.

\section{Agradecimientos}

Los autores agradecen al CONCYTEC por el apoyo económico recibido para la compra del material biológico utilizado en este estudio.

Asimismo agradecen la colaboración del Ing. Jaime Angeles en las salidas para la obtención del material biológico y al M.Sc. Francisco Pinilla por su apoyo en el trabajo de laboratorio, y revisión del manuscrito.

\section{Literatura citada}

Acuña E. \& Cid L.1995. On the ecology of two sympatric flounders of the genus Paralichthys in the Bay of Coquimbo, Chile. Netherlands Journal of Sea Research. 34 (1-3): 7-18

Ahlstrom E.H, Amaoka D.A., Moser H.G. \&. Sumida B. 1984. Pleuronectiformes: Development. In: H. G. Moser, editor. Ontogeny and systematics of fishes. American Society of Ichthyologists and Herpetologist. Special publication. $\mathrm{N}^{\circ} 1$ : 640-670.

Chinchayán M., Vera G., Cisneros R. \& Carrera L. 1997. Notas sobre el cultivo de los lenguados Paralichthys adspersus y Etropus ectenes en ambiente controlado. Inf. Prog. Inst. Mar. Perú. 64:34-51.

Chirichigno N. 1974. Informe 44 "Clave para identificar los peces marinos del Perú". Instituto del Mar del Perú. Callao.

Coll J. 1991. Acuicultura Marina Animal. Ediciones MundiPrensa. Madrid.

Copaira M. \& Montalvo C. 1972. Dimorfismo sexual en el paiche (Arapaima gigas, Cuvier, 1829). Rec. Inv. Pec. (IVITA). Universidad Nacional Mayor de San Marcos. 1(2): 203-207.

Flores M., Vera S., Marcelo R. \& Chirinos E. 1996. Estadísticas de los desembarques de la pesquería marina peruana 1992-1993-1994. Instituto del Mar del Perú. Informe $\mathrm{N}^{\mathrm{o}} 118$

1997.

Estadísticas de los desembarques de la pesquería marina peruana 1995-1996. Instituto del Mar del Perú. Informe $\mathrm{N}^{\circ} 129$.

1998a.

Estadísticas de los desembarques de la pesquería marina peruana 1970-1982 Instituto del Mar del Perú. Informe $\mathrm{N}^{\circ} 134$.

1998b.

Estadísticas de los desembarques de la pesquería marina peruana 1996-1997. Instituto del Mar del Perú. Informe No 140.
Estadísticas de los desembarques de la pesquería marina peruana 1998. Instituto del Mar del Perú. Informe $\mathrm{N}^{\mathrm{o}}$ 152.

$2000 b$.

Estadísticas de los desembarques de la pesquería marina peruana 1999. Instituto del Mar del Perú. Informe $\mathrm{N}^{\circ}$ 155.

Huet M. 1983. Tratado de piscicultura. Ediciones MundiPrensa. Madrid.

Jones A. 1974. Sexual maturity, fecundity and growth of the turbot Scophthalmus maximus L. Jounal of Marine Biological Association U. K. 54: 109-125.

Mendo J. 1987. Contribución a la edad y crecimiento de una especie de cachema Cynoscion analis de la zona frente a Paita. Boletín Instituto del Mar del Perú. 11(1):1-39.

Pauly D. \& Munro J. 1984. Once more on the comparison of growth in fish and invertebrates. Fishbyte. 2(1): 21.

Ramos J. 1982. Contribución al estudio de la sexualidad del lenguado Solea solea (Linneo, 1758) (Pisces, Soleidae). Inv. Pesq. 46(2): 275-286.

Ramos J. \& Ramos E. 1985. Estudio del dimorfismo sexual en el lenguado, Solea solea (Linneo, 1758) (Pisces, Soleidae), de las costas de Castellón (Mediterráneo occidental). Investigación Pesquera. 49(4): 537-544.

Samamé M. \& Castañeda J. 1999. Biología y pesquería del lenguado Paralichthys adspersus, con especial referencia al área norte del litoral peruano, departamento de Lambayeque. Boletín Instituto del Mar del Perú. 18 (1-2): 15-48.

Silva A. 1990. Experiencias sobre la inducción al desove del lenguado (Paralichthys microps, Gunther, 1881) con hormona G.C.H. Red de Acuicultura, Bogotá (Colombia). Boletín 4 (1):8-10.

1994. Spawning of the chilean flounder Paralichthys microps_(Gunther ,1881) in captivity. Journal of the World Aquaculture Society. 25(2): 342344.

2001. Advanced in the culture research of smalleye flounder, Paralichthys microps, and Chilean flounder, $P$. adspersus in Chile. Journal of Applied Aquaculture. 11 (1-2):147-164.

\& Flores H. 1989. Consideraciones sobre el desarrollo y crecimiento larval del lenguado (Paralichthys adspersus Steindachner, 1887) cultivado en laboratorio. Revista Pacífico Sur (Número Especial).

Sparre P., Ursin E. \& Venema S.C. 1989. Introduction to tropical fish assessment. Part 1. Manual. FAO. Fisheries Technical Paper Nº306/1. Roma.

Tresierra A., Carvajal W., Oliva J. \&. Culquichicon Z. 1984. Apuntes de biología pesquera. Parte I. Trujillo (Perú).

Vásquez E., Fernández-Pato, Martínez-Tapia C., Blanco G. \& Sánchez Prado J.A. 2002. Evolución de las tasas de crecimiento en individuos diploides y triploides de rodaballo Scophthalmus maximus (L., 1758). Boletín Instituto Español de Oceanografía. 18 (1-4):239-243.

Zar J.M. 1984. Biostatistical analysis. Second Edition. Prentice-Hall. Englewood Cliffs, New Jersey.

\footnotetext{
${ }^{1}$ Facultad de Pesquería, Universidad Nacional Agraria La Molina, Av. La Universidad s/n, Apartado 456. Lima 12-Perú, bangeles@lamolina.edu.pe, angeles_pinilla@yahoo.com

${ }^{2}$ Facultad de Pesquería, Universidad Nacional Agraria La Molina, Av. La Universidad s/n, Apartado 456. Lima 12-Perú,jmendo@lamolina.edu.pe
} 